\title{
Structural, optical, photoluminescence, dielectric and electrical studies of vacuum-evaporated CdTe thin films
}

\author{
ZIAUL RAZA KHAN, M ZULFEQUAR and MOHD. SHAHID KHAN* \\ Department of Physics, Jamia Millia Islamia, New Delhi 110 025, India
}

MS received 18 September 2010

\begin{abstract}
Highly-oriented CdTe thin films were fabricated on quartz and glass substrates by thermal evaporation technique in the vacuum of about $2 \times 10^{-5}$ torr. The CdTe thin films were characterized by X-ray diffraction (XRD), UV-VIS-NIR, photoluminescence spectroscopy and scanning electron microscopy (SEM). X-ray diffraction results showed that the films were polycrystalline with cubic structure and had preferred growth of grains along the (111) crystallographic direction. Scanning electron micrographs showed that the growth of crystallites of comparable size on both the substrates. At the room temperature, photoluminescence spectra of the films on both the substrates showed sharp peaks with a maximum at $805 \mathrm{~nm}$. This band showed significant narrowing suggesting that it originates from the transitions involving grain boundary defects. The refractive index of CdTe thin films was calculated using interference pattern of transmission spectra. The optical band gap of thin films was found to allow direct transition with energy gap of 1.47-1.50 eV. a.c. conductivity of CdTe thin films was found to increase with the increase in frequency whereas dielectric constant was observed to decrease with the increase in frequency.
\end{abstract}

Keywords. CdTe; optical properties; photoluminescence; SEM; a.c. conductivity.

\section{Introduction}

Cadmium telluride (CdTe) is an important group II-VI semiconductor with a direct band gap of $1.5 \mathrm{eV}$ at room temperature. Extensive research was done in the last two decades on CdTe thin films, mainly due to its potential applications, particularly in the field of polycrystalline thin film solar cells and also large area electronic devices like fieldeffect transistors, radiation detector, optical thin film filters, nonlinear integrated optical devices, light emitting diodes (LEDs) and laser heterostructures for emission in the infrared spectral range (Goldsmid et al 1980; Patel and Patel 1992; Nair et al 1993; Cruz et al 1999; Romeo et al 2001; Van Gheluwe et al 2005). The absorption edge in CdTe is very sharp due to its direct energy band gap and thus efficiency of absorption of light is very high around $90 \%$. The maximum theoretical efficiency reported for CdTe solar cells is $29 \%$. Whereas the conversion efficiency of CdTe solar cell was reported up to $16.5 \%$ (Kosyachenko et al 2009). Still, the conversion efficiency, crystallites size, optical properties and conduction phenomena in CdTe thin films are some open-ended problems demanding extensive research. The growth of high-quality and low-cost CdTe polycrystalline thin films for producing high conversion efficiency solar cells is the current topic of research. $\mathrm{CdTe} / \mathrm{CdS}$ solar cells are one of the prospective candidates for widespread commercial

\footnotetext{
*Author for correspondence (mskhan@jmi.ac.in)
}

success in solar energy conversion and also for use as a substrate for the important infrared detector material $\mathrm{HgCdTe}$, dilute magnetic semiconductor, epitaxial layers and quantum well structures (Morales-Acevedo 2006; Robin et al 2009).

A number of techniques of deposition were adopted to obtain CdTe thin films (Feldman et al 1981; Gongoi and Barua 1982; Anthony et al 1984; Matsumoto et al 1984; Takahashi et al 1984; Wang and Reinhard 1984; Matsumura et al 1985; Gayle and Biancaniello 1995). All these techniques have their own merits and demerits in producing high-quality CdTe films. Among various other techniques, thermal evaporation techniques are simple and economical. Recently structural and optical properties of thermallyevaporated CdTe polycrystalline thin films were reported (Lalitha et al 2007; Al-Ghamdi et al 2010), however the work on important parameters like nucleation of grain size, microstructures and how to affect various properties of polycrystalline CdTe thin films is still to be done (Bacaksiz et al 2007; Semaltianos et al 2009). In the present work, the microstructural, optical, dielectric and electrical, and photoluminescence properties of CdTe thin films fabricated on glass and quartz substrates were investigated.

\section{Experimental}

Analytical grade CdTe powder with $99.99 \%$ purity was purchased from Alfa Aesar (UK). CdTe thin films were fabricated by thermal evaporation technique in a vacuum about $2 \times 10^{-5}$ torr, using vacuum coating unit. Molybdenum boat 
was used as the evaporation source and quartz and glass substrates were placed directly above the source at a distance of nearly $15 \mathrm{~cm}$. The quartz and glass substrates were cleaned with freshly prepared acetone, detergent solution and distilled water, respectively. Further, the substrates were subjected to ultrasonic cleaning prior to the evaporation of CdTe. The transmittance spectra for as-deposited CdTe thin films on glass and quartz substrates were obtained in the range of 190-2500 nm using JASCO UV-VIS-NIR spectrometers (Model-V570). The structure of the films was studied using Philips analytical diffractometer (Model PW3710). The Photo luminescence (PL) measurement at an excitation wavelength of $525 \mathrm{~nm}$ was carried out using an Ar-ion laser with the maximum output power of $200 \mathrm{~mW}$. The surface morphology of CdTe thin films was performed by the JSM6700 scanning electron microscope. The dielectric properties like dielectric constant $\left(\varepsilon_{\mathrm{r}}\right)$ and loss tangent $(\tan \delta)$ of the films were calculated from the a.c. measurements of impedence, capacitance and phase angle measured as a function of frequency using agilent impedence analyser (model-4294A).

\section{Results and discussion}

\subsection{X-ray diffraction analysis}

XRD patterns of CdTe thin films on different substrates are shown in figure 1 , whereas the XRD pattern of CdTe powder are presented in figure 2. XRD analysis of the thin films indicated the cubic structure with peaks at $2 \theta=23.51^{\circ}$, $39.20^{\circ}$ and $46 \cdot 18^{\circ}$ with the orientations ( $\left(\begin{array}{lll}1 & 1 & 1\end{array}\right),\left(\begin{array}{lll}2 & 2 & 0\end{array}\right)$ and (3 11 1), respectively. It was observed that the CdTe crystallites have similar growth on quartz and glass substrates along (1 11 1) index. For the calculation of grain size and strain of CdTe thin films on glass and quartz substrates, we have used Williamson method, which was first proposed by Williamson and Hall and is customarily referred as Williamson Hall method. The equation for the separation of crystallites size and the strain takes the following form (Semaltianos et al 1995):

$$
\beta \cos \theta=\lambda / D_{\mathrm{v}}+4 \varepsilon(\sin \theta),
$$
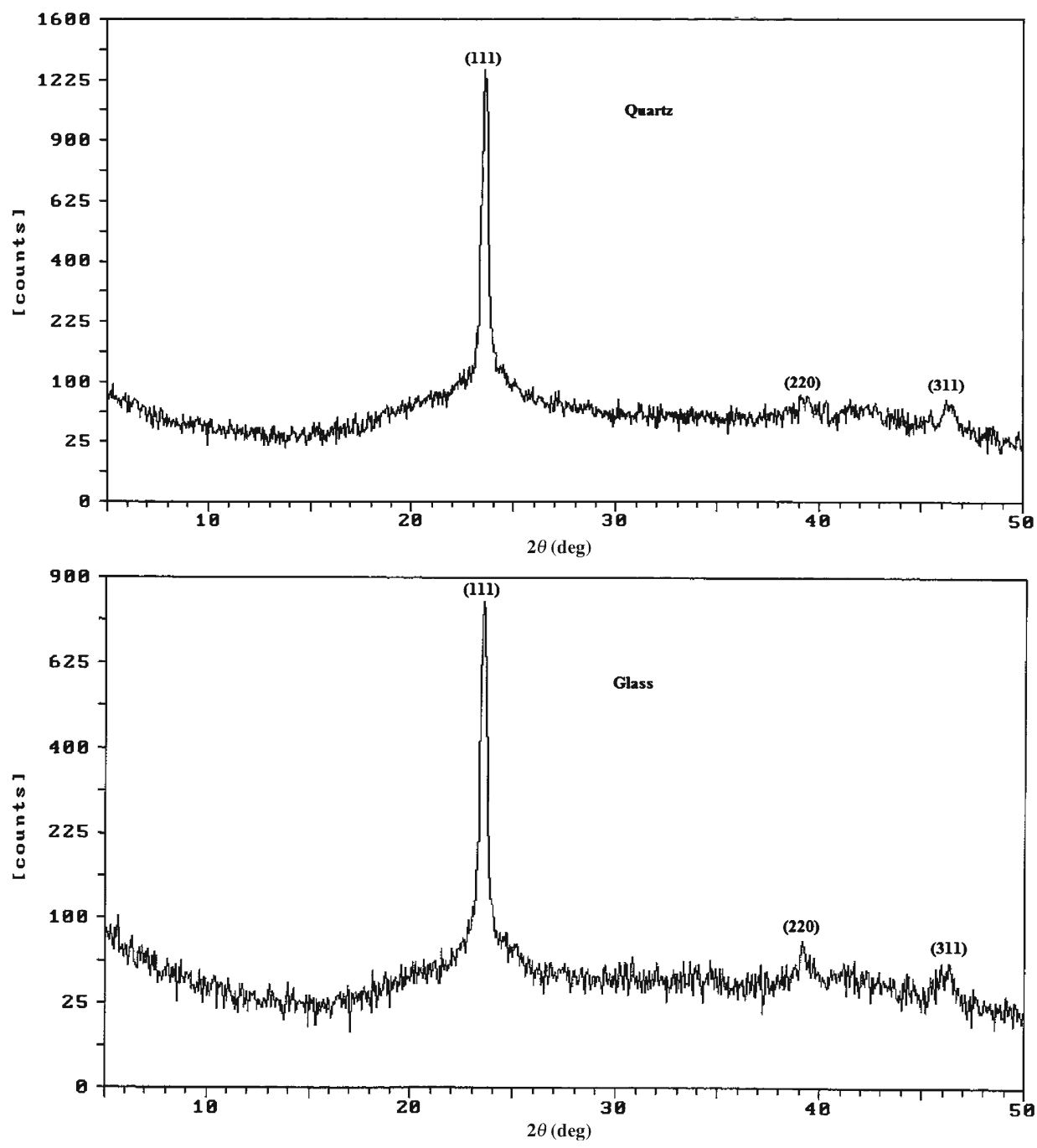

Figure 1. XRD patterns of CdTe thin films on glass and quartz substrates. 


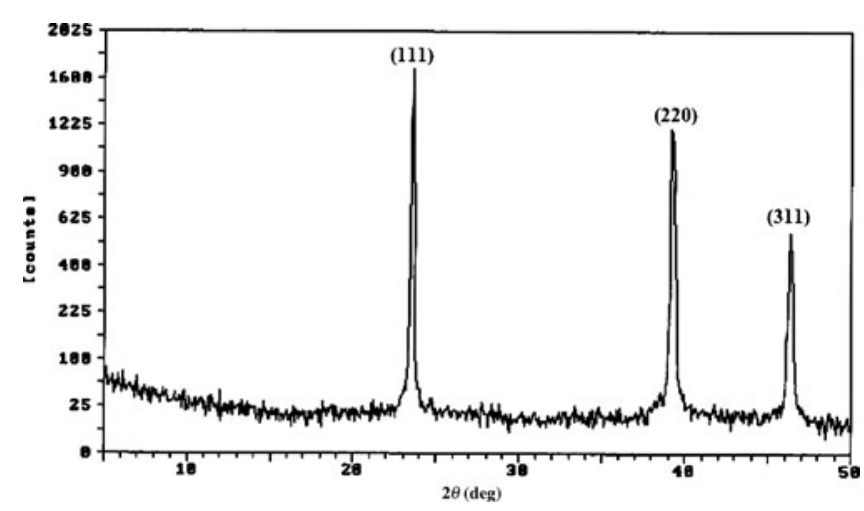

Figure 2. XRD pattern of CdTe powder.

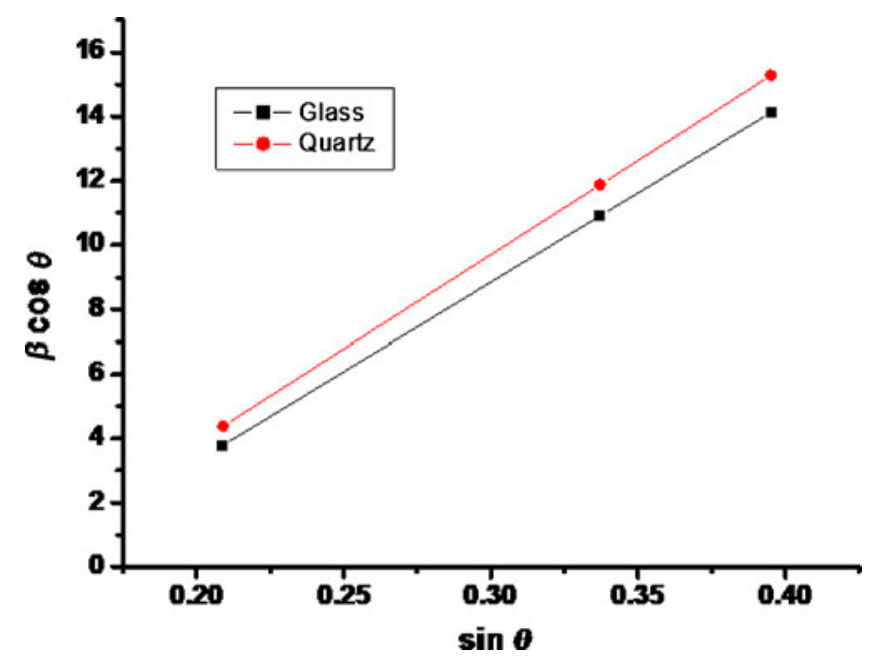

Figure 3. Grain size and strain calculated using Williamson-Hall method.

where $\lambda$ is the wavelength of X-ray used ( $1.54 \AA$ in our case) and $D_{v}, \varepsilon$ are the grain size and strain, respectively.

The peak width at half maximum $\beta$ of each reflection to be used in (1) is calculated using relation (Shaaban et al 2009):

$$
\beta=B\left(1-b^{2} / B^{2}\right) \text { (rad.), }
$$

where $B$ and $b$ are the width of the same Bragg-peak from the XRD pattern of thin film and CdTe powder, respectively. The plots $\beta \cos \theta$ vs $\sin \theta$ for the film on glass and quartz substrates are shown in figure 3 . The values of grain size and strain are calculated from ordinate-intersection and slope of the graph, respectively. The values of width, grain size and strain of each reflection of CdTe thin films on different substrates are given in table 1, from which it was observed that the strain is slightly more in films on quartz substrates than on the glass substrates.

\subsection{Optical properties}

Optical properties of CdTe thin films on glass and quartz substrates are discussed in this section. Transmission spectra
Table 1. Comparative view of breadth, grain size and strain of CdTe thin films on glass and quartz.

\begin{tabular}{|c|c|c|c|c|c|c|}
\hline \multirow[b]{2}{*}{ Substrates } & \multicolumn{3}{|c|}{$\beta$} & \multirow{2}{*}{$\begin{array}{c}\text { Grain } \\
\text { size }(\mathrm{nm})\end{array}$} & \multirow{2}{*}{ Strain } & \multirow{2}{*}{$\begin{array}{c}\text { Band- } \\
\text { gap }(e V)\end{array}$} \\
\hline & $(111)$ & $(220)$ & (311) & & & \\
\hline Glass & 0.00209 & $0 \cdot 01158$ & 0.01669 & 45 & $0 \cdot 0138$ & 1.50 \\
\hline Quartz & 0.00448 & $0 \cdot 01266$ & $0 \cdot 01664$ & 56 & 0.0143 & 1.47 \\
\hline
\end{tabular}

of CdTe thin films are shown in figure 4. The transmission of the thin films in the spectral range of $1000-2500 \mathrm{~nm}$ is found to be very high. This confirms that the thin films have semiconducting properties; as it is an established fact that the pure semiconducting compounds have a sharp absorption edge (Nowak 1995). The well-known relation near the fundamental absorption edge is given by

$$
\alpha=A\left(h v-E_{\mathrm{g}}\right)_{h v}^{m}
$$

where $A$ is the characteristic parameter (independent of photon energy) for this transition, $h$ the Plank's constant, $v$ the frequency of light, $E_{\mathrm{g}}$ the band gap and $m$ the parameter which characterizes the transition process involved. The parameter $m$ takes the values of 2 and $1 / 2$ for the direct allowed transition and for indirect allowed transition, respectively. In the case of CdTe polycrystalline thin films on different substrates the direct transitions are valid. The plots $(\alpha h v)^{2}$ vs $h v$ for the polycrystalline CdTe thin films on glass and quartz substrates are shown in figure 5 . The values of energy band gap of the thin films as determined by extrapolation of linear portion of the plots $(\alpha h v)^{2}$ against $h v$ to the energy axis. The calculated band gap for both the films is found to be in the range of $1.47-1.50 \mathrm{eV}$, which is in good agreement with its reported values for the CdTe thin films (Khairnar et al 2003). The energy band gap value of CdTe thin films on glass substrates is found to be more than that of the film deposited on quartz substrate.

The refractive index is determined using the method of recording envelopes around the interference maxima and minima in the transmittance spectra (Swanepoel 1983). Following Swanepoel method, the value of refractive index at a particular wavelength can be calculated using the expression:

$$
n=\left[N+\left(N-S^{2}\right)^{1 / 2}\right]^{1 / 2},
$$

where

$$
N=2 S \frac{T_{\max }-T_{\min }}{T_{\max } T_{\min }}+\frac{S^{2}+1}{2},
$$

where $T_{\max }$ and $T_{\min }$, are the transmittance maximum and the corresponding minimum at a certain wavelength $\lambda$. On the other hand, the necessary values of the refractive index of the substrate are obtained from the transmittance spectrum of the 


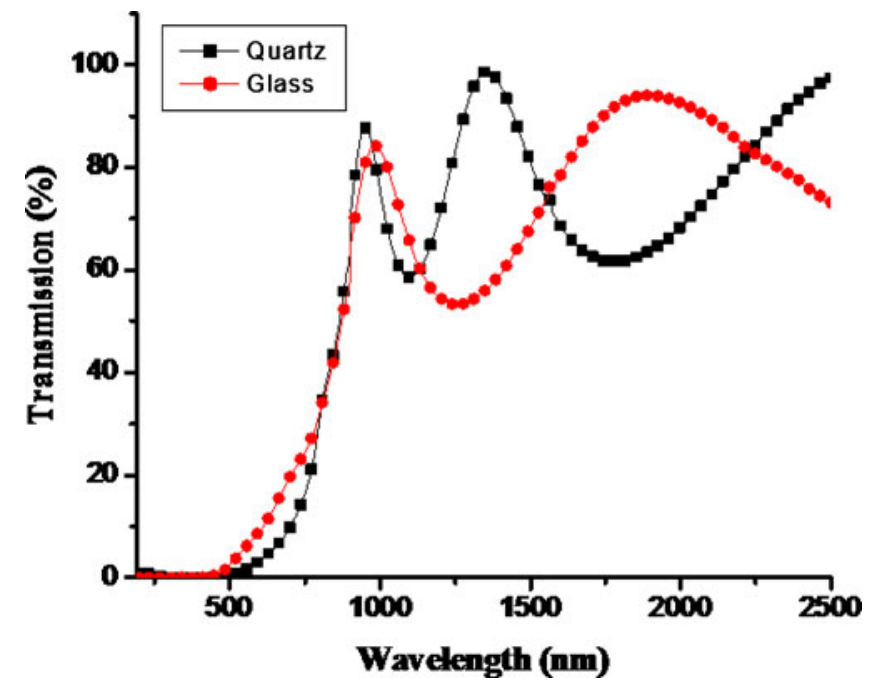

Figure 4. Transmission spectra of CdTe thin films on glass and quartz.

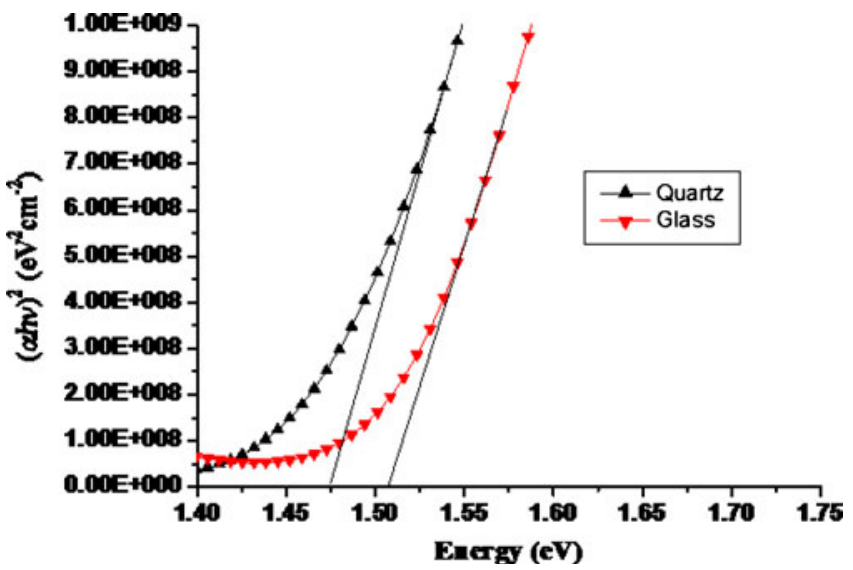

Figure 5. $(\alpha h v)^{2}$ vs $h v$ plots.

substrate, $T_{\mathrm{s}}$ using the well known equation (Rakhshani and Al-Azab 2000):

$$
S=\frac{1}{T_{\mathrm{s}}}+\left(\frac{1}{T_{\mathrm{s}}}-1\right)^{1 / 2} .
$$

The values of the refractive index, $n$ for CdTe polycrystalline thin films as calculated from (4) are plotted against the wavelength in figure 6. It is observed from these graphs of $n$ vs $\lambda$ that the refractive index, $n$ decreases with the increase in the wavelength, $\lambda$.

\subsection{Photoluminescence}

Photoluminescence spectra of CdTe polycrystalline thin films on glass and quartz are shown in figure 7. The photoluminescence spectroscopy plays a very important role to determine the exact energy levels of nanostructures or poly- crystalline thin films. In PL spectra of CdTe films, a strong and narrow emission peak at $805 \mathrm{~nm}$ is observed when the sample is excited at $532 \mathrm{~nm}$, showing no trap-related emission. The photoluminescence spectra of CdTe thin films on quartz and glass substrates differ in peaks width (FWHM). The luminescence at $805 \mathrm{~nm}$ may be attributed to a higher level transition in CdTe crystallites. The significant narrowing of the band may be due to the growth of round grains shaped with the average size of $50-100 \mathrm{~nm}$ and it may involve transitions associated with grain-boundary defects as reported by Marafi et al (2003). It was reported that this kind of band-edge luminescence arises from the recombination of exciton and/or shallow trapped electron-hole pairs (Armani et al 2007). The film grown on quartz substrates has high peak width $\sim 60 \mathrm{meV}$ (FWHM) whereas the film grown on glass substrates has low peak width $\sim 10 \mathrm{meV}$. This difference may be due to the microstructure of the thin films.

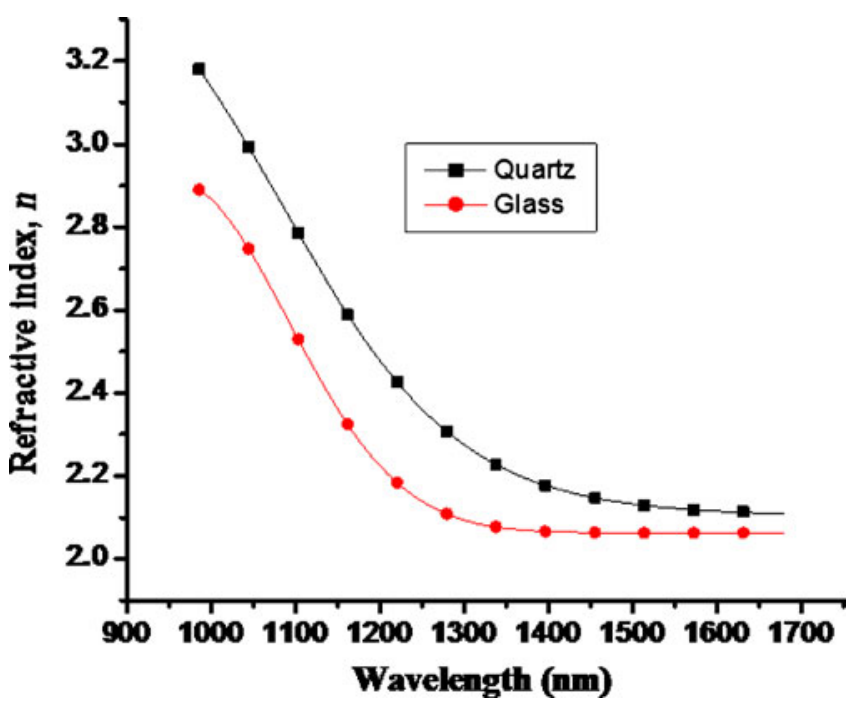

Figure 6. Refractive index of CdTe thin films.

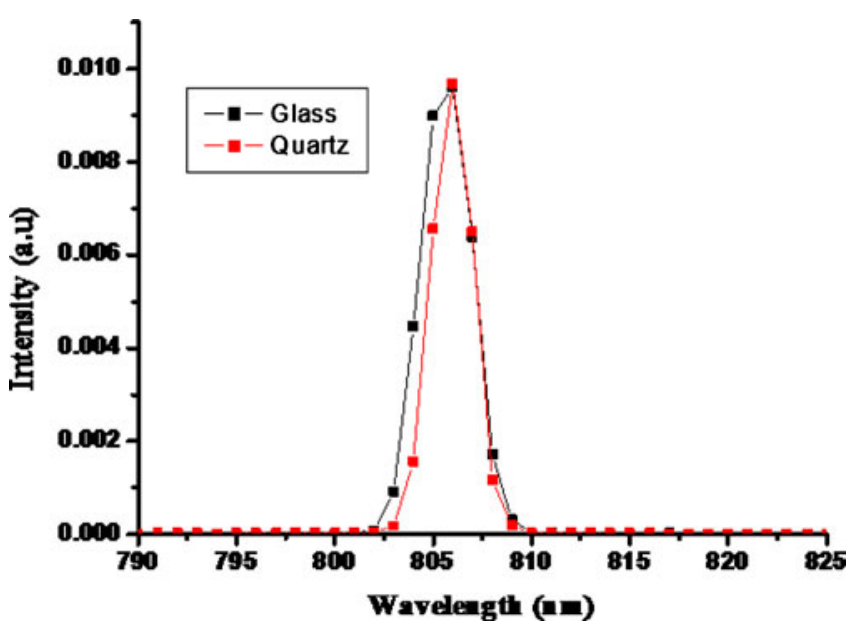

Figure 7. Photoluminescence spectra of CdTe thin films on glass and quartz substrates. 


\subsection{SEM analysis}

Surface morphology of thermally-evaporated CdTe thin films on quartz and glass substrates are shown in figures 8(a), (b). From these SEM micrographs, it is evident that there is small difference in the grain size of films grown on quartz and glass substrates. The substrates are well covered by large round shaped CdTe crystallites of average grain size of 50-100 nm, similar to that obtained using the XRD analysis. Microstructure of CdTe thin films on quartz and glass substrates shows non-uniform growth of grains as was reported by Sharma et al (2005).

\subsection{Dielectric and electrical properties}

The variations of dielectric constant, dielectric loss, $\tan \delta$ and a.c. conductivity with frequency for the CdTe thin films in the frequency range of $10 \mathrm{~Hz}-100 \mathrm{kHz}$ are shown in figures 9(a)-(c), respectively. The dielectric constant is found to decrease with the increase in frequency. The thin films of CdTe exhibited the similar behaviour in the case of dielectric loss. This type of behaviour suggests a distribution of relaxation times in the CdTe thin films system. The XRD
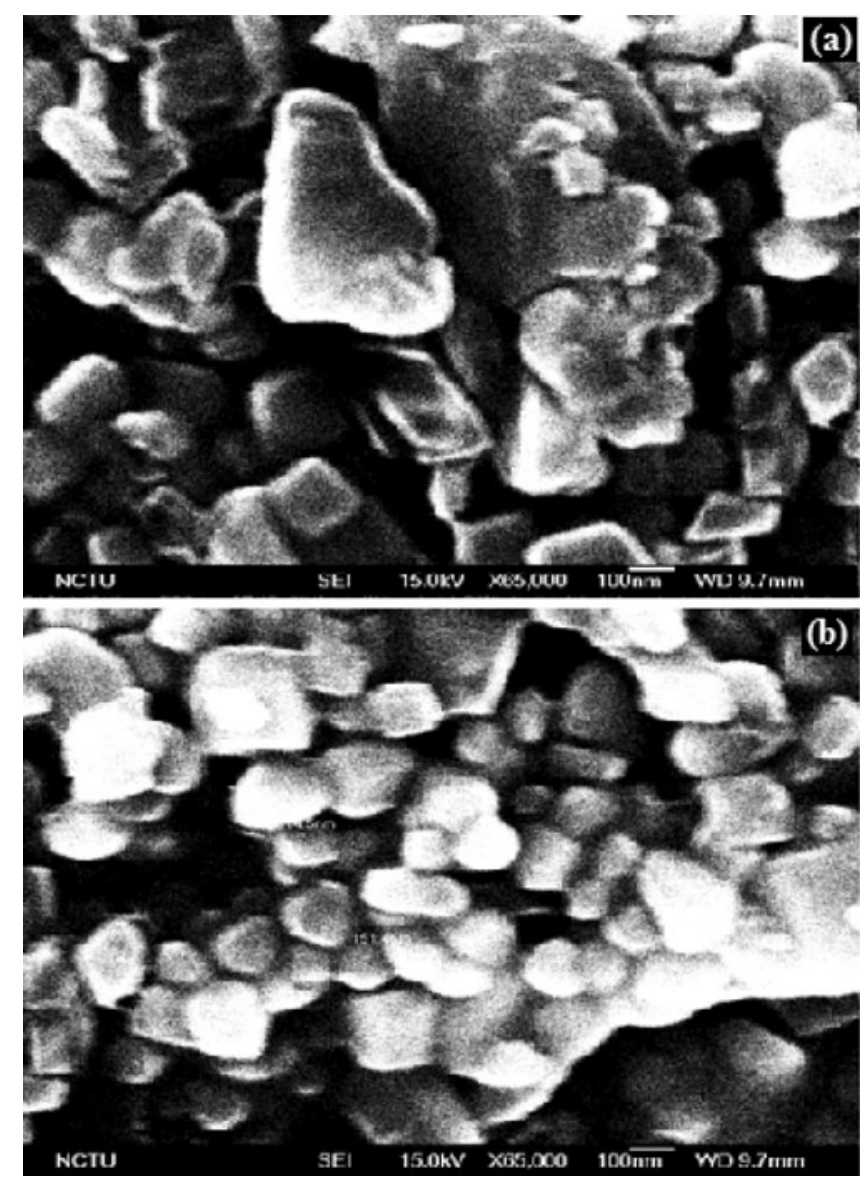

Figure 8. SEM micrograph of CdTe thin films on (a) quartz and (b) glass substrates.
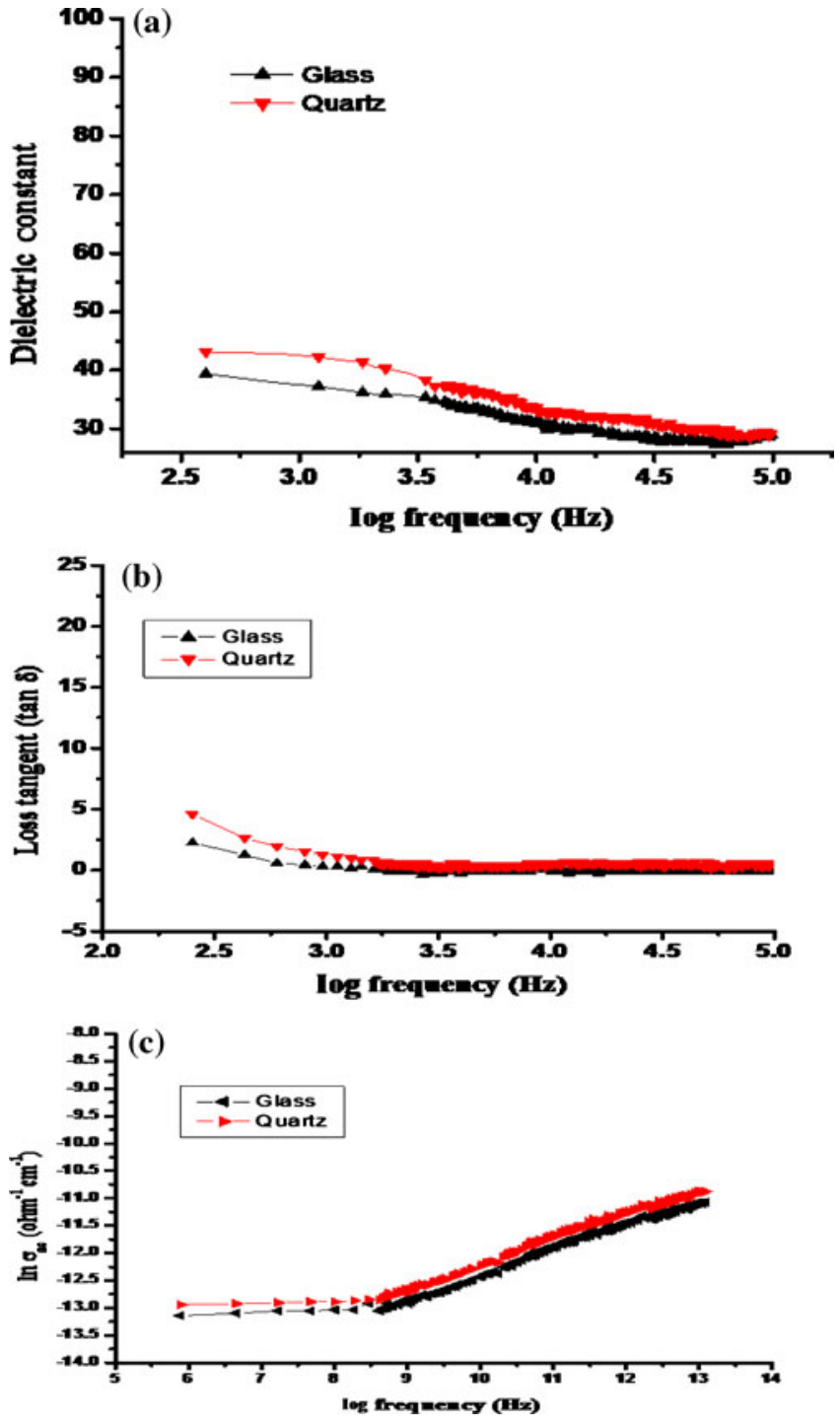

Figure 9. Variations of (a) dielectric constant, (b) loss tangent and (c) a.c. conductivity with frequency.

patterns of thin films have only one intense peak suggesting the noticeable disordered nature of the system, the same is also confirmed from the SEM micrographs. Structural information of thin films suggests simple or multifaceted defects such as defect centres, micro voids, imperfections, cracks, etc that may be present in the films. These defects play very important role in transport phenomena, as these defects originate the defect states like shallow and deep states which may act as donor or acceptors. Such type of states affect the transport phenomena in thin films to a great extent by acting as traps and recombination centres for carriers. These states may also lead to the probable formation of dipoles, which are responsible for the dielectric behaviour. When the thin films are subjected to electric field, the electron hop between localized states. Transport of charge carriers between these sites, hop from a donor to an acceptor state. As a result, each pair of sites forms a dipole and contributes to distributed dielectric relaxation (Prabakar et al 2002). 
The a.c. conductivity is determined using the relation,

$$
\sigma_{\mathrm{ac}}=\varepsilon_{0} \varepsilon_{\mathrm{r}} \omega \tan \delta .
$$

Figure 9(c) displays the variation of a.c. conductivity with frequency. A.c. conductivity of CdTe thin films is found to increase with the frequency. Such type of behaviour is shown commonly by various other materials and was reported in the literature (Mardare and Rusu 2004). The increase in a.c. conductivity with frequency again indicates the existence of localized conduction in CdTe thin films in which the a.c. conductivity increases with increase in frequency. In contrast to this, in the case of free band conduction the a.c. conductivity decreases with increase in frequency (Anwar and Hogarth 1990).

\section{Conclusions}

The device-grade CdTe polycrystalline thin films were fabricated by the thermal evaporation technique on quartz and glass substrates. The XRD studies have revealed that the thin films are polycrystalline in nature and CdTe crystallites have cubic structure. The structural parameters like grain size and strain were calculated from XRD pattern. Transmission of CdTe thin films is found to be $85-90 \%$ in infrared and near infrared region. Energy band gap of thin films is found to be in the range of $1.47-1.50 \mathrm{eV}$. Refractive index of thin films is very high in infrared region and it is found to decrease gradually with increasing wavelength. SEM studies revealed the columnar growth of CdTe crystallites. Photoluminescence spectra of CdTe thin films showed sharp peaks around $805 \mathrm{~nm}$. Overall investigation suggests that CdTe polycrystalline thin films on quartz and glass substrates have similar properties.

\section{Acknowledgements}

The authors are thankful to Dr Tariqul Islam and Dr Shakeb Khan, Department of Electrical Engineering, Jamia Millia, for extending Impedance Analyzer Facility for electric and dielectric studies of samples.

\section{References}

Al-Ghamdi A A, Khan S A, Nagat A and Abdel-Sadek M S 2010 Opt. Laser Technol. 421181

Anthony T C, Fahrenbruch A L and Bube R H 1984 J. Vac. Sci. Technol. A2 1296
Anwar M and Hogarth C A 1990 J. Mater. Sci. 253906

Armani N, Salviati G, Nasi L, Bosio A, Mazzamuto S and Romeo N 2007 Thin Solid Films 5156184

Bacaksiz E, Basol B M, Altunbas M, Novruzov V, Yanmaz E and Nezir S 2007 Thin Solid Films 5153079

Cruz L R, Kazmerski L L, Moutinko H R, Hasoon F, Dhere R G and de Avillez R 1999 Thin Solid Films 35044

Feldman B J, Boone J L and Van Doren T V 1981 Appl. Phys. Lett. 38703

Gayle F W and Biancaniello F S 1995 Nanostruct. Mater. 6429

Goldsmid H J, Giutronich J E and Kalia M M 1980 Sol. Energy 24 435

Gongoi S and Barua K 1982 Thin Solid Films 92227

Khairnar U P, Bhavsar D S, Vaidya R U and Bhavsar G P 2003 Mater. Chem. Phys. 80421

Kosyachenko L A, Sabchuk A I and Grushko E V 2009 Thin Solid Films $\mathbf{5 1 7} 2386$

Lalitha S, Karazhanov S Zh, Ravindran P, Senthilarasu S, Sathyamoorthy R and Janabergenov J 2007 Physica B 387227

Marafi M, El-Akkad F and Pradeep B 2003 J. Mater. Sci.-Mater. Electron. 1421

Mardare D and Rusu G I 2004 J. Optoelectron. Adv. Mater. 6333

Matsumoto H, Kuribayashi K, Uda H, Kamatsu Y, Nakano A and Ikegami S 1984 Sol. Cells 11367

Matsumura N, Ohshima T, Saraie J and Yodogawa Y 1985 J. Cryst. Growth 71361

Morales-Acevedo A 2006 Sol. Energy 80675

Nair M T S, Nair P K, Pathirana H M K K, Zingaro R A and Meyers E A 1993 J. Electrochem. Soc. 1402987

Nowak M 1995 Thin Solid Films 266258

Patel N G and Patel P G 1992 Solid-State Electrochem. 351269

Prabakar K, Narayandass Sa K and Mangalaraj D 2002 Cryst. Res. Technol. 371094

Rakhshani A E and Al-Azab A S 2000 J. Phys. Condens. Matter 12 8745

Robin I C, Taupin M, Derone R, Solignac A, Ballet P and Lusson A 2009 Appl. Phys. Lett. 95202104

Romeo A, Bätzner D L, Zogg H, Vignali C and Tiwari A N 2001 Sol. Energ. Mater. Sol. Cells 67311

Semaltianos N G, Karczewski G, Hu B, Wojtowicz T and Furdyna J K 1995 Phys. Rev. B24 17499

Semaltianos N G, Logothetidis S, Perrie W, Romani S, Potter R J, Sharp M, Dearden G and Watkins K G 2009 Appl. Phys. Lett. 95 033302

Shaaban E R, Afifyb N and El-Taher A 2009 J. Alloys Compd. 482 400

Sharma R K, Rastogi A C, Jain K and Singh G 2005 Physica B 36680

Swanepoel R 1983 J. Phys. Sci. Instrument. 161214

Takahashi M, Uosaki K and Kita H 1984 J. Appl. Phys. 553879

Van Gheluwe J, Versluys J, Poelman D and Clauws P 2005 Thin Solid Films 480264

Wang F and Reinhard D K 1984 J. Appl. Phys. 553702 